\title{
LIPSCHITZ NORMALLY EMBEDDED SET AND TANGENT CONES AT INFINITY
}

\author{
LUIS RENATO G. DIAS AND NILVA RODRIGUES RIBEIRO
}

\begin{abstract}
We prove that any analytic set in $\mathbb{C}^{n}$ with a unique tangent cone at infinity is an algebraic set. We prove that the degree of a complex algebraic set in $\mathbb{C}^{n}$, which is Lipschitz normally embedded at infinity, is equal to the degree of its tangent cone at infinity.
\end{abstract}

\section{INTRODUCTION}

This note is motivated by the arguments presented in [6].

Let $X$ be a path connected subset of $\mathbb{C}^{n}$. Given $x, y \in X$, we set the inner distance on $X$ between $x$ and $y$, denoted by $d_{X}(x, y)$, as the infimum of the length $(\gamma)$ where $\gamma$ varies on the set of continuous paths on $X$ connecting $x$ to $y$. We recall the following definition from [2].

Definition 1.1. A path connected subset $X$ of $\mathbb{C}^{n}$ is called Lipschitz normally embedded if there exists a positive real number $C$ such that

$$
d_{X}(x, y) \leq C\|x-y\|,
$$

for all $x, y \in X$.

Lipschitz normal embedding and necessary conditions for a set to be Lipschitz normally embedded have been studied by many authors; see for instance [1, 4, 5, 9, 10, 11] and the references cited therein.

In a recent work, Fernandes and Sampaio [6] introduced the following definition in the global case.

Definition 1.2. A path connected subset $X$ of $\mathbb{C}^{n}$ is called Lipschitz normally embedded at infinity if there exists a compact subset $K \subset X$ such that $X \backslash K$ is Lipschitz normally embedded.

When a pure dimensional analytic set $X \subset \mathbb{C}^{n}$ has a unique tangent cone at infinity, we denote it by $C_{\infty}(X)$ and call it the tangent cone of $X$ at infinity; see Definitions 2.6 and 2.7 for details. The main theorem of [6] is the following one:

Date: March 23, 2021.

2020 Mathematics Subject Classification. 58K30, 14B05.

Key words and phrases. Lipschitz geometry, tangent cone at infinity, analytic set, algebraic set.

The first author was partially supported by the Fapemig-Brazil Grant APQ-00431-14 and CNPq-Brazil grants 401251/2016-0 and 304163/2017-1. 
Theorem 1.3 ([ᄄ], Theorem 1.1]). Let $X \subset \mathbb{C}^{n}$ be a closed and pure d-dimensional analytic subset. Suppose $X$ has a unique tangent cone at infinity and this cone is a d-dimensional complex linear subspace of $\mathbb{C}^{n}$. If $X$ is Lipschitz normally embedded at infinity, then $X$ is an affine linear subspace of $\mathbb{C}^{n}$.

Let $V \subset \mathbb{C}^{n}$ be an algebraic set. Bearing in mind that $\operatorname{deg} V=1$ if and only if $V$ is a linear subspace of $\mathbb{C}^{n}$; see for instance Proposition 3.3 of [6]. We prove in this note that Theorem 1.3 holds true with the following more general statement.

Theorem 1.4. Let $X \subset \mathbb{C}^{n}$ be a closed and pure s-dimensional analytic subset. Suppose $X$ has a unique tangent cone at infinity and this cone is a pure $k$-dimensional complex algebraic set of $\mathbb{C}^{n}$. Then $X$ is an algebraic set and $s=k$. Moreover, if $X$ is Lipschitz normally embedded at infinity, then $\operatorname{deg} X=\operatorname{deg} C_{\infty}(X)$.

We remark that from equality (2.1) of [7], it follows that $\operatorname{deg} X \geq \operatorname{deg} C_{\infty}(X)$ is always true for any pure dimensional complex algebraic sets $X$ and $C_{\infty}(X)$.

This note is organized as follows. In section 2, we recall the definition of algebraic region after [12] and the definition of tangent cones at infinity. In Theorem 2.9, we prove that if an unbounded set $V \subset \mathbb{C}^{n}$ has a unique tangent cone at infinity, we say $C_{\infty}(V)$, and this cone lies in an algebraic region, then there exists an algebraic region $\Omega$ such that $V$ and $C_{\infty}(V)$ lie in $\Omega$.

As a consequence of Theorem 2.9, it follows that $\operatorname{dim} X=\operatorname{dim} C_{\infty}(X)$ for any complex algebraic set (Proposition 2.10). This last result can be seen as a version at infinity of a result of Whitney on tangent cones at a point of an analytic variety.

In Section 3, we recall the definition of degree of a complex algebraic set and we present the proof of Theorem 1.4. The proof is motivated by the arguments of [6].

Finally, we observe that a local version of Theorem 1.4 follows from Corollary 3.13 and Remark 3.8 of [5]. Precisely, let $X \subset \mathbb{C}^{m}$ be a pure analytic set and $0 \in X$. We say that $X$ is Lipschitz normally embedded at 0 if there exists a neighborhood $U \subset \mathbb{C}^{m}$ of 0 such that $X \cap U$ is Lipschitz normally embedded. The tangent cone of $X$ at 0 is denoted by $C(X, 0)$; see for instance section 8.1 of [3]. We denote by $\mu_{0}(X)$ and $\mu_{0}(C(X, 0))$ the multiplicity at 0 of $X$ and $C(X, 0)$ respectively; see for instance section 11 of [3]. Thus, a local version of Theorem 1.4 is the next result, whose proof follows from Corollary 3.13 and Remark 3.8 of [5].

Theorem 1.5. Let $X \subset \mathbb{C}^{m}$ be a pure dimensional complex analytic set with $0 \in X$. If $X$ is a Lipschitz normally embedded set at 0 , then $\mu_{0}(X)=\mu_{0}(C(X, 0))$.

We point out that another proof of Theorem 1.5 may be given by similar argument of the proof of Theorem 1.4

\section{Algebraic Region And tangent CONE AT infinity}

2.1. Algebraic region. We start by recalling the definition of algebraic region after [12]. 
Definition 2.1 (See p. 672 of [12]). Let $n \geq 2$. A set $\Omega \subset \mathbb{C}^{n}$ is called an algebraic region of type $(k, n)$ if there exist vector subspaces $V_{1}, V_{2}$ in $\mathbb{C}^{n}$ and positive real numbers $A, B$ such that the following conditions hold: $\operatorname{dim} V_{1}=k, \operatorname{dim} V_{2}=n-k, \mathbb{C}^{n}=V_{1}+V_{2}$ and $\Omega$ consists of those $z \in \mathbb{C}^{n}$ for which:

$$
\left\|z^{\prime \prime}\right\| \leq A\left(1+\left\|z^{\prime}\right\|\right)^{B}
$$

where $z=z^{\prime}+z^{\prime \prime}$, with $z^{\prime} \in V_{1}$ and $z^{\prime \prime} \in V_{2}$.

A geometric characterization of analytic sets of $\mathbb{C}^{n}$ which are algebraic is given by the next theorem.

Theorem 2.2 (See Theorem 2 of [12]). A closed analytic subset $V \subset \mathbb{C}^{n}$ of pure dimension $k$ is algebraic if and only if $V$ lies in some algebraic region $\Omega$ of type $(k, n)$.

Let $\Omega \subset \mathbb{C}^{n}$ be an algebraic region of type $(k, n)$, with $V_{1}, V_{2} \subset \mathbb{C}^{n}$ and constants $A, B$ as in Definition 2.1. We may choose linear coordinates $(x, y)$ in $\mathbb{C}^{n}$ such that $x \in V_{1}$ and $y \in V_{2}$. In this coordinate system, we denote by $\pi_{\Omega}$ the canonical projection from $\mathbb{C}^{n}$ to $V_{1}$, i.e., $\pi_{\Omega}(x, y)=x$. In the proof of Proposition 2.4 we need a fact concerning the projection $\pi_{\Omega}$, as follows.

Lemma 2.3. If $V$ is a closed set in $\mathbb{C}^{n}$ such that $V \subset \Omega$, then the restriction $\pi_{\Omega}: V \rightarrow V_{1}$ is a proper mapping.

Proof. Let $\left\{z_{j}\right\}_{j \in \mathbb{N}}$ be any sequence in $V$ with $\lim _{j \rightarrow \infty}\left\|z_{j}\right\|=\infty$. We have to show that $\lim _{j \rightarrow \infty}\left\|\pi_{\Omega}\left(z_{j}\right)\right\|=\infty$. We may write $z_{j}=\left(x_{j}, y_{j}\right)$, with $x_{j} \in V_{1}$ and $y_{j} \in V_{2}$. By hypothesis, $\left(x_{j}, y_{j}\right)$ satisfies the inequality (11) and, therefore, $\lim _{j \rightarrow \infty}\left\|x_{j}\right\|=\infty$. This finishes the proof, since $\pi_{\Omega}\left(z_{j}\right)=x_{j}$.

Let $V \subset \mathbb{C}^{n}$ be an algebraic set. It follows from [12, p. 677] that if $\operatorname{dim} V \leq k$, then $V$ lies in an algebraic region of type $(k, n)$. The converse is also true, by the next result.

Proposition 2.4. Let $V$ be an algebraic set in $\mathbb{C}^{n}$. If $V$ lies in an algebraic region of type $(k, n)$, then $\operatorname{dim} V \leq k$.

Proof. Let $\Omega \subset \mathbb{C}^{n}$ be an algebraic region of type $(k, n)$, with $V_{1}, V_{2}$ in $\mathbb{C}^{n}$ and constants $A, B$ as in Definition 2.1 and that $V \subset \Omega$. From Lemma 2.3, the projection $\pi_{\Omega}: V \rightarrow V_{1}$ is a proper mapping. Then $\operatorname{dim} V=\operatorname{dim} \pi_{\Omega}(V)$; see for instance Propositions 1 and 2 of [3, p. 31]. Since $\operatorname{dim} V_{1}=k$, it follows that $\operatorname{dim} V \leq k$.

2.2. Tangent cone at infinity. Let us start this section recalling two equivalent definitions of tangent cone at infinity of algebraic sets after Theorem 1 of [13].

Definition 2.5. Let $V$ be an algebraic set. The geometric tangent cone $C_{g, \infty}(V)$ of $V$ at infinity is defined by the set of tangent vectors of $V$ at infinity, in the sense that $v \in C_{g, \infty}(V)$ if and only if there exist sequences $\left\{x_{j}\right\}_{j \in \mathbb{N}} \subset V,\left\{t_{j}\right\}_{j \in \mathbb{N}} \subset \mathbb{C}$, such that $\lim _{j \rightarrow \infty}\left\|x_{j}\right\|=\infty$ and $\lim _{j \rightarrow \infty} t_{j} x_{j}=v$. 
The algebraic tangent cone $C_{a, \infty}(V)$ of $V$ at infinity is defined by the set

$$
C_{a, \infty}(V)=\left\{v \in \mathbb{C}^{n} \mid f^{*}(v)=0 \text { for all } f \in I(V)\right\}
$$

where $I(V)$ denotes the ideal defining $V$, and for each polynomial $f \in I(V), f^{*}$ denotes its homogeneous component of highest degree.

It follows by Theorem 1 of [13] that $C_{g, \infty}(V)=C_{a, \infty}(V)$ for any algebraic set $V \subset \mathbb{C}^{n}$. In particular, Theorem 1 of [13] can be seen as a version at infinity of a well-known theorem of $\mathrm{H}$. Whitney [15] on tangent cones at a point of an analytic variety.

For any unbounded subset $V$ of $\mathbb{C}^{m}$, it is defined in [6] the tangent vectors at infinity with respect to a sequence of real positive numbers, as follows.

Definition 2.6. Let $V \subset \mathbb{C}^{n}$ be an unbounded subset. Given a sequence of real positive numbers $\left\{t_{j}\right\}_{j \in \mathbb{N}}$ such that $\lim _{j \rightarrow \infty} t_{j}=\infty$, we say that $v \in \mathbb{C}^{n}$ is tangent to $V$ at infinity with respect to $\left\{t_{j}\right\}_{j \in \mathbb{N}}$ if there exists a sequence of points $\left\{x_{j}\right\}_{j \in \mathbb{N}} \subset V$ such that $\lim _{j \rightarrow \infty} \frac{1}{t_{j}} x_{j}=v$.

Definition 2.7. Let $V \subset \mathbb{C}^{n}$ be an unbounded subset and $T=\left\{t_{j}\right\}_{j \in \mathbb{N}}$ be a sequence of real positive numbers such that $\lim _{j \rightarrow \infty} t_{j}=\infty$. Denote by $E_{T}(V)$ the set of $v \in \mathbb{C}^{n}$ which are tangent to $V$ at infinity with respect to $T$. We call $E_{T}(V)$ a tangent cone of $V$ at infinity. When $V$ has a unique tangent cone at infinity, we denote it by $C_{\infty}(V)$ and we call $C_{\infty}(V)$ the tangent cone of $V$ at infinity.

In general, the problem of determining the uniqueness of tangent cones at infinity for unbounded sets is a difficult problem. For instance, a conjecture by Meeks [14, Conjecture 3.15] states that any embedded minimal surface in $\mathbb{R}^{3}$ with quadratic area growth has a unique tangent cone at infinity. See for instance [8] for a partial answer of this conjecture.

REMARK 2.8. Let $X \subset \mathbb{C}^{n}$ be an unbounded algebraic set. From Corollary 2.16 of [6], we know that $X$ has an unique tangent cone at infinity and, with Theorem 1 of [13], $C_{\infty}(X)=C_{a, \infty}(X)=C_{g, \infty}(X)$. See also Proposition 2.21 of [6]. In particular, if $v \in C_{\infty}(X)$ then $\lambda v \in C_{\infty}(X)$, for any $\lambda \in \mathbb{R}$.

Theorem 2.9. Let $V \subset \mathbb{C}^{n}$ be an unbounded subset. Suppose that $V$ has a unique tangent cone at infinity and this cone lies in an algebraic region $\tilde{\Omega}$ of type $(k, n)$. Then there exists an algebraic region $\Omega$ of type $(k, n)$ such that $V$ and $C_{\infty}(V)$ lie in $\Omega$.

Proof. It follows from Definition 2.1 that there exist vector spaces $V_{1}$ and $V_{2}$ in $\mathbb{C}^{n}$, with $\operatorname{dim} V_{1}=k, \operatorname{dim} V_{2}=n-k, \mathbb{C}^{n}=V_{1}+V_{2}$, and positive real numbers $\tilde{A}, B$ such that for any $z \in \tilde{\Omega}$ (hence, in particular, for $z \in C_{\infty}(V)$ ) we have:

$$
\left\|z^{\prime \prime}\right\|<\tilde{A}\left(1+\left\|z^{\prime}\right\|\right)^{B}
$$

where $z=z^{\prime}+z^{\prime \prime}$, with $z^{\prime} \in V_{1}$ and $z^{\prime \prime} \in V_{2}$. Since $(1+t)^{s} \leq(1+t)$, for any $t \geq 0$ and $0 \leq s \leq 1$, we may assume in (2) that $B \geq 1$.

We claim that there exists a positive real number $R$ such that, for any $w \in V$, we have:

$$
\left\|w^{\prime \prime}\right\|<R\left(1+\left\|w^{\prime}\right\|\right)^{B}
$$


where $w=w^{\prime}+w^{\prime \prime}$, with $w^{\prime} \in V_{1}$ and $w^{\prime \prime} \in V_{2}$.

If the claim is not true, this means that there exists a sequence $\left\{w_{j}\right\}_{j \in \mathbb{N}} \subset V$ such that $\left\|w_{j}^{\prime \prime}\right\|>k\left(1+\left\|w_{j}^{\prime}\right\|\right)^{B}$ and, up to a subsequence, we may suppose that $\lim _{j \rightarrow \infty} \frac{w_{j}^{\prime \prime}}{\left\|w_{j}^{\prime \prime}\right\|}=y_{0} \in$ $V_{2}$, with $\left\|y_{0}\right\|=1$. It follows that $\lim _{j \rightarrow \infty}\left\|w_{j}^{\prime \prime}\right\|=\infty$ and therefore $\lim _{j \rightarrow \infty}\left\|w_{j}\right\|=\infty$ since $\mathbb{C}^{n}$ is a direct sum of $V_{1}$ and $V_{2}$. We have

$$
\frac{1}{k}>\frac{\left(1+\left\|w_{j}^{\prime}\right\|\right)^{B}}{\left\|w_{j}^{\prime \prime}\right\|} \geq \frac{\left(1+\left\|w_{j}^{\prime}\right\|\right)}{\left\|w_{j}^{\prime \prime}\right\|} \geq \frac{\left\|w_{j}^{\prime}\right\|}{\left\|w_{j}^{\prime \prime}\right\|} .
$$

Let $t_{j}:=(\tilde{A})^{-1}\left\|w_{j}^{\prime \prime}\right\|$. We have $\lim _{j \rightarrow \infty} t_{j}=\infty$ and

$$
\lim _{j \rightarrow \infty} \frac{1}{t_{j}} w_{j}=\lim _{j \rightarrow \infty} \frac{1}{t_{j}}\left(w_{j}^{\prime}+w_{j}^{\prime \prime}\right)=\tilde{A} y_{0} .
$$

It follows that $\tilde{A} y_{0} \in C_{\infty}(V)$ by Definition 2.7. Now, since $V_{2}$ is a linear space and $y_{0} \in V_{2}$, we have $\tilde{A} y_{0} \in\left(V_{2} \cap C_{\infty}(V)\right)$. This is a contradiction, since (2) does not hold for $\tilde{A} y_{0}$.

Therefore, if follows that there exists a positive real number $R$ such that for any $w \in V$ we have:

$$
\left\|w^{\prime \prime}\right\|<R\left(1+\left\|w^{\prime}\right\|\right)^{B}
$$

where $z=w^{\prime}+w^{\prime \prime}$, with $w^{\prime} \in V_{1}$ and $w^{\prime \prime} \in V_{2}$. Let $A:=\max \{R, \tilde{A}\}$, then $V$ and $C_{\infty}(V)$ lie in an algebraic region $\Omega$ of type $(k, n)$, with vector spaces $V_{1}$ and $V_{2}$ in $\mathbb{C}^{n}$, with $\operatorname{dim} V_{1}=k, \operatorname{dim} V_{2}=n-k$ and positive real numbers $A$ and $B$. This finishes the proof.

Bearing in mind that $C_{\infty}(V)=C_{a, \infty}(V)=C_{g, \infty}(V)$ for any unbounded algebraic set $V \subset \mathbb{C}^{n}$ (see Remark 2.8) and that, by definition of dimension, $\operatorname{dim}_{\mathbb{R}}(V)=2 \operatorname{dim}_{\mathbb{C}}(V)$, we finish this section with the following result.

Proposition 2.10. Let $V \subset \mathbb{C}^{n}$ be an unbounded algebraic set. Then $\operatorname{dim} V=\operatorname{dim} C_{\infty}(V)$.

Proof. It follows from Corollary 2.18 of [6] that $\operatorname{dim} C_{\infty}(V) \leq \operatorname{dim} V$.

On the other hand, let $k=\operatorname{dim} C_{\infty}(V)$, which is an algebraic set. By [12, page 677], $C_{\infty}(V)$ lie in an algebraic region $\tilde{\Omega}$ of type $(k, n)$ and by Theorem $\left[2.9, V\right.$ and $C_{\infty}(V)$ lie in a common algebraic region $\Omega$ of type $(k, n)$. Then, it follows from Proposition 2.4 that $\operatorname{dim} V \leq k=\operatorname{dim} C_{\infty}(V)$.

The previous result can be seen as a version at infinity of a result of Whitney on tangent cones at point of an analytic variety; see for instance Lemma 8.11 of [15].

\section{PROOF OF MAIN THEOREM}

We recall the definition of degree of an algebraic set. We follow section 11.3 of [3]. The complex projective space is denoted by $\mathbb{P}^{n}$ and $\mathbb{C}^{n}$ is identified with the open set $\left\{\left[x_{0}: x_{1}: \ldots: x_{n}\right] \in \mathbb{P}^{n} \mid x_{0} \neq 0\right\}$ of $\mathbb{P}^{n}$ through the mapping $\tau: \mathbb{C}^{n} \rightarrow \mathbb{P}^{n}, \tau\left(x_{1}, \ldots, x_{n}\right)=$ $\left[1: x_{1}: \ldots: x_{n}\right]$. 
Definition 3.1. Let $X \subset \mathbb{C}^{n}$ be an algebraic set. The degree of $X$, denoted by $\operatorname{deg} X$, is the degree of its closure in $\mathbb{P}^{n}$.

In the proof of Theorem 1.4, we consider the following. Let $X \subset \mathbb{C}^{n}$ be a pure $k$ dimensional algebraic set. We have that $X$ has a unique tangent cone at infinity and it is an algebraic set; see Remark 2.8. From Proposition 2.10 and Theorem 2.9, there exists an algebraic region $\Omega$ of type $(k, n)$ such that $X$ and $C_{\infty}(X)$ lie in $\Omega$. Then, there exist vector spaces $V_{1}, V_{2} \subset \mathbb{C}^{n}$ and constants $A, B$ as in Definition 2.1. We may choose linear coordinates $(x, y)$ in $\mathbb{C}^{n}$ such that $x \in V_{1}$ and $y \in V_{2}$. In this coordinate system, we denote by $\pi_{\Omega}$ the canonical projection from $\mathbb{C}^{n}$ to $V_{1}$, i.e., $\pi_{\Omega}(x, y)=x$. Moreover, we may assume that $V_{1}$ and $V_{2}$ are orthogonal to each other and that $\pi_{\Omega}$ is an orthogonal projection; see Theorem 3 of page 78 of [3]. By similar argument as in proof of Lemma 2.3, it follows that the closures of $X$ and $V_{2}$ (respectively, the closures of $C_{\infty}(X)$ and $V_{2}$ ) in $\mathbb{P}^{n}$ do not have points at infinity in common. Then, it follows by Corollary 1 of [3, p. 126] the following fact concerning the projection $\pi_{\Omega}$ :

Lemma 3.2. The restriction $\pi_{\Omega}: X \rightarrow V_{1}$ (respectively, $\pi_{\Omega}: C_{\infty}(X) \rightarrow V_{1}$ ) is a ramified covering with number of sheets equal to $\operatorname{deg} X$ (respectively, $\operatorname{deg} C_{\infty}(X)$ ).

3.1. Proof of Theorem 1.4. First, we prove that $X$ is an algebraic set and that $\operatorname{dim} X=$ $\operatorname{dim} C_{\infty}(X)$. By Theorem 2.2. $C_{\infty}(X)$ lies in an algebraic region of type $(k, n)$. It follows from Theorem 2.9 that $X$ and $C_{\infty}(X)$ lie in an algebraic region $\Omega$ of type $(k, n)$. Then, by Theorem 2.2 , we have that $X$ is an algebraic set. That $\operatorname{dim} X=\operatorname{dim} C_{\infty}(X)$, it follows by Proposition 2.10 .

Now, we assume that $X$ is Lipschitz normally embedded at infinity. Thus, there exist a compact subset $K \subset X$ and a positive real number $C$ such that

$$
d_{X}\left(x_{1}, x_{2}\right) \leq C\left\|x_{1}-x_{2}\right\| \text {, for all } x_{1}, x_{2} \in X \backslash K \text {. }
$$

We denote $\operatorname{deg} X=d_{1}$ and $\operatorname{deg} C_{\infty}(X)=d_{2}$.

From Theorem 2.9 and Proposition 2.10 , there exists an algebraic region $\Omega$ of type $(k, n)$ containing $X$ and $C_{\infty}(X)$. We may consider $\Omega$ as in the paragraph before Lemma 3.2. Thus, there exist vector spaces $V_{1}$ and $V_{2}$ in $\mathbb{C}^{n}$, with $\operatorname{dim} V_{1}=k, \operatorname{dim} V_{2}=n-k$, $\mathbb{C}^{n}=V_{1}+V_{2}$, and positive real numbers $A, B$ such that for any $z \in\left(X \cup C_{\infty}(X)\right)$ the following holds:

$$
\left\|z^{\prime \prime}\right\|<A\left(1+\left\|z^{\prime}\right\|\right)^{B}
$$

where $z=z^{\prime}+z^{\prime \prime}$, with $z^{\prime} \in V_{1}$ and $z^{\prime \prime} \in V_{2}$.

Let $\pi_{\Omega}: \mathbb{C}^{n} \rightarrow V_{1}$ be the projection. As in the paragraph before Lemma 3.2, we may assume that $V_{1}, V_{2}$ are orthogonal to each other and that $\pi_{\Omega}$ is an orthogonal projection.

It follows by Lemma 3.2 that the restriction $\pi_{\Omega}: X \rightarrow V_{1}$ (respectively, $\pi_{\Omega}: C_{\infty}(X) \rightarrow$ $V_{1}$ ) is a ramified covering with number of sheets equal to $d_{1}$ (respectively, $d_{2}$ ).

\footnotetext{
${ }^{1}$ here, the phrase "at infinity" means the set $\mathbb{P}^{n} \backslash \mathbb{C}^{n}$.
} 
Let $\Sigma_{1} \subset V_{1}$ and $\Sigma_{2} \subset V_{1}$ be the ramification locus of the restriction of $\pi_{\Omega}$ to $X$ and to $C_{\infty}(X)$ respectively.

We have that $\Sigma_{i}$ is a complex algebraic set such that $\operatorname{dim} \Sigma_{i}<k$, for $i=1,2$. Then, there exists a vector $v \in V_{1} \backslash\left(C_{\infty}\left(\Sigma_{1}\right) \cup C_{\infty}\left(\Sigma_{2}\right)\right)$. In particular, for big enough $t \in \mathbb{R}_{>0}$, we have $t v \notin\left(\Sigma_{1} \cup \Sigma_{2}\right)$.

Let $\lambda_{1}(t), \ldots, \lambda_{d_{1}}(t) \in X$ be the different liftings of $t v$ by $\pi_{\Omega}$ restricted to $X$, i.e., $\pi_{\Omega}\left(\lambda_{i}(t)\right)=t v, i=1, \ldots, d_{1}$.

Since $\mathbb{C}^{n}=V_{1}+V_{2}$, we may write $\lambda_{i}(t)=t v+y_{i}(t)$, with $y_{i}(t) \in V_{2}$, for $i=1, \ldots, d_{1}$.

We have that $\frac{\left\|y_{i}(t)\right\|}{t}$ is bounded when $t \rightarrow \infty$, for $i=1, \ldots, d_{1}$. Otherwise, there exists an index $i \in\left\{1, \ldots, d_{1}\right\}$ and there exists a sequence of positive numbers $\left\{t_{l}\right\}_{l \in \mathbb{N}}$ such that $\frac{\left\|y_{i}\left(t_{l}\right)\right\|}{t_{l}}>l$, for any $l$. We may assume that $\lim _{l \rightarrow \infty} \frac{y_{i}\left(t_{l}\right)}{\left\|y_{i}\left(t_{l}\right)\right\|}=w_{i}$, with $w_{i} \in V_{2}$. It follows that

$$
\lim _{l \rightarrow \infty} \frac{A}{\left\|y_{i}\left(t_{l}\right)\right\|} \lambda_{i}\left(t_{l}\right)=A \lim _{l \rightarrow \infty} \frac{t_{l} v+y_{i}\left(t_{l}\right)}{\left\|y_{i}\left(t_{l}\right)\right\|}=0+A w_{i}
$$

It follows by definition of $C_{\infty}(X)$ and (6) that $A w_{i} \in C_{\infty}(X)$ and, therefore, $A w_{i} \in$ $\left(C_{\infty}(X) \cap V_{2}\right)$. This contradicts $C_{\infty}(X) \subset \Omega$.

Therefore, for $i \in\left\{1, \ldots, d_{1}\right\}$, we have that $\frac{\left\|y_{i}(t)\right\|}{t}$ is bounded when $t \rightarrow \infty$. Thus, we may assume that there exists a sequence of real positive numbers $\left\{t_{j}\right\}_{j \in \mathbb{N}}$ such that $\lim _{j \rightarrow \infty} t_{j}=\infty$ and, for $i=1, \ldots, d_{1}, \lim _{j \rightarrow \infty} \frac{y_{i}\left(t_{j}\right)}{t_{j}}=v_{i}$, with $v_{i} \in V_{2}$.

Then,

$$
\lim _{j \rightarrow \infty} \frac{1}{t_{j}} \lambda_{i}\left(t_{j}\right)=\lim _{j \rightarrow \infty}\left(\frac{t_{j} v}{t_{j}}+\frac{y_{i}\left(t_{j}\right)}{t_{j}}\right)=v+v_{i}, \text { for } i=1, \ldots, d_{1} .
$$

By definition of $C_{\infty}(X)$, we have $v+v_{i} \in C_{\infty}(X)$, for $i=1, \ldots, d_{1}$. It follows that the curves $\beta_{i}(t)=t\left(v+v_{i}\right)$ lie in $C_{\infty}(X)$, for $i=1, \ldots, d_{1}$; see Remark 2.8. Therefore, $\beta_{1}, \ldots, \beta_{d_{1}}$ are liftings of $t v$ by $\pi_{\Omega}$ restricted to $C_{\infty}(X)$, i.e., $\pi_{\Omega}\left(\beta_{i}(t)\right)=t v, i=1, \ldots, d_{1}$.

If we suppose that $d_{1}>d_{2}$, then there exists two distinct indexes $s_{1}, s_{2} \in\left\{1, \ldots, d_{1}\right\}$ such that $\beta_{s_{1}}=\beta_{s_{2}}$, which implies $v_{s_{1}}=v_{s_{2}}$ by definitions of $\beta_{s_{1}}$ and $\beta_{s_{2}}$. Then, by (7), it follows that

$$
\lim _{j \rightarrow \infty} \frac{1}{t_{j}}\left(\lambda_{s_{1}}\left(t_{j}\right)-\lambda_{s_{2}}\left(t_{j}\right)\right)=0 .
$$

Since $v \in V_{1} \backslash\left(C_{\infty}\left(\Sigma_{1}\right) \cup C_{\infty}\left(\Sigma_{2}\right)\right)$, it follows from Lemma 2.4 of [13] that there exists $\delta>0$ such that, for $j$ big enough, the following hold:

$$
\operatorname{dist}\left(t_{j} v, \Sigma_{1}\right)>\delta t_{j}
$$

Now, we follow Lemma 2.2 of [4] and proof of Theorem 1.1 of [6]. For any path $\omega$ in $X$ joining $\lambda_{s_{1}}\left(t_{j}\right)$ to $\lambda_{s_{2}}\left(t_{j}\right)$, the path $\pi_{\Omega}(\omega)$ is a loop in $V_{1}$ with base point $t_{j} v$ which is not contractible in $V_{1} \backslash \Sigma_{1}$. Then, with (9), it follows that the length of $\pi_{\Omega}(\omega)$ is at least $2 \delta t_{j}$. Now, since $\pi_{\Omega}$ is an orthogonal projection, it follows that 


$$
d_{X}\left(\lambda_{s_{1}}\left(t_{j}\right), \lambda_{s_{2}}\left(t_{j}\right)\right) \geq 2 \delta t_{j}
$$

Then, by (41) and (10), we have:

$$
\frac{1}{t_{j}} C\left\|\lambda_{s_{1}}\left(t_{j}\right)-\lambda_{s_{2}}\left(t_{j}\right)\right\| \geq \frac{1}{t_{j}} d_{X}\left(\lambda_{s_{1}}\left(t_{j}\right), \lambda_{s_{2}}\left(t_{j}\right)\right) \geq 2 \delta, \text { for } j \text { big enough. }
$$

From (8) and (11), as $j \rightarrow \infty$, it follows the following contradiction:

$$
0 \geq 2 \delta
$$

Therefore, we have $d_{1} \leq d_{2}$. Now, as mentioned in the Introduction, $d_{1} \geq d_{2}$ is always true for any pure dimensional complex algebraic sets $X$ and $C_{\infty}(X)$; see equality (2.1) of [7. This ends the proof.

\section{REFERENCES}

[1] Birbrair, L. And Fernandes, A., Inner metric geometry of complex algebraic surfaces with isolated singularities, Comm. Pure Appl. Math., 61, (2008), 1483-1494.

[2] Birbrair, L. And Mostowski, T., Normal embeddings of semialgebraic sets, Michigan Math. J. 47 (2000), 125-132.

[3] Chirka, E. M., Complex Analytic Sets. Kluwer Academic Publishers, Dordrecht (1989).

[4] Denkowski, M. and TibăR, M., Testing Lipschitz non-normally embedded complex spaces, Bull. Math. Soc. Sci. Math. Roumanie (N.S.) 62 (2019), 93-100.

[5] Fernandes, A. And Sampaio, J. E., Tangent cones of Lipschitz normally embedded sets are Lipschitz normally embedded. Appendix by Anne Pichon and Walter D. Neumann, Int. Math. Res. Not. IMRN (2019), n. 15, 4880-4897.

[6] Fernandes, A. And SAmpaio, J. E., On Lipschitz rigidity of complex analytic sets, J. Geom. Anal 30 (2020), 706-718.

[7] Fernández de Bobadilla, J. and Fernandes, A. and Sampaio, J. E., Multiplicity and degree as bi-Lipschitz invariants for complex sets, J. Topol. 11 (2018), 958-966.

[8] Gallagher, P., A criterion for uniqueness of tangent cones at infinity for minimal surfaces, J. Geom. Anal. 29 (2019), 370-377.

[9] Kerner, D. and Pedersen, H. M. and Ruas, M. A. S., Lipschitz normal embeddings in the space of matrices, Math. Z., 290 (2018), 485-507.

[10] Neumann, W. D. and Pedersen, H. M. and Pichon, A., A characterization of Lipschitz normally embedded surface singularities, J. Lond. Math. Soc. (2) 101 (2020), 612-640.

[11] Neumann, W. D. and Pedersen, H. M. and Pichon, A., Minimal surface singularities are Lipschitz normally embedded, J. Lond. Math. Soc. (2), 101 (2020), 641-658.

[12] Rudin, W., A geometric criterion for algebraic varieties, J. Math. Mech. 17 (1967/1968), 671683.

[13] LÊ, C. T. and Pham, T. S., On tangent cones at infinity of algebraic varieties, Journal of Algebra and its Applications 17 (2018), 1850143-1 - 1850143-10.

[14] Meeks, III, W. H., Global problems in classical minimal surface theory, In: D. Hoffman (ed.), Global Theory of Minimal Surfaces, pp. 453-470. American Mathematical Society, Providence, RI (2005).

[15] Whitney, H., Tangents to an analytic variety, Ann. of Math. 81 (1965), 496-549. 
Universidade Federal de Uberlândia, Faculdade de Matemática, Av. João Naves de Ávila 2121, 1F-153 - CEP: 38408-100, UBerlÂNDIA, Brazil.

Email address: lrgdias@ufu.br

Universidade Federal de Viçosa, Campus Rio Paranaíba, Km 7, Zona Rural, MG - 230 Rodoviário - CEP: 38810-000, Rio PARANAíba, BraziL.

Email address: nilva.ribeiro@ufv.br 